\title{
Preformed metal crowns may last longer than fillings
}

\author{
Are preformed metal crowns (PMC) more effective than conventional filling \\ materials (amalgam, composite, glass ionomers and compomers) for the \\ restoration of primary teeth?
}

\author{
Innes NPT, Ricketts DNJ, Evans DJP. Preformed metal crowns for \\ decayed primary molar teeth. Cochrane Database of Systematic \\ Reviews 2007; issue 1
}

Data sources Searches were made using the Cochrane Central Register of Controlled Trials (CENTRAL), Medline, Embase and the System for Information on Grey Literature in Europe (SIGLE).

Study selection Randomised controlled trials (RCT) were chosen that assessed the effectiveness of PMC, compared with filling materials or in children where there had been no treatment of tooth decay in one or more primary molar teeth.

Data extraction and synthesis Forty-seven records were retrieved by the search strategies, some of which proved to be duplicates. Ultimately, 14 studies were scrutinised. None met the inclusion criteria and six studies were excluded from the review because they were either retrospective in design or they reported prospective outcomes but were not randomised.

Conclusions No RCT were available for appraisal. Although the use of PMC is recommended in clinical practice by the British Society of Paediatric Dentistry (BSPD), the evidence to support this is not strong, consisting mainly of case reports and uncontrolled studies. The lower quality levels of evidence that have been produced nevertheless have some strength, since clinical outcomes are consistently in favour of PMC even though many studies analyse casts placed on the most damaged of the pair of teeth. It is important that the absence of evidence for PMC is not misinterpreted as evidence for their lack of efficacy.

\section{Commentary}

Several clinical guidelines recommend the use of PMC for restoring extensive carious lesions in primary molars, yet this systematic review has found that good quality evidence is not available to support the effectiveness of this intervention. Is there a paradox here? Not at all. This review intended to summarise only the results of high-quality RCT: a clinical guideline summarises all available evidence. In fact, if we were to base our practice only on treatments with strong evidence of effectiveness, we would need to stop performing about threequarters of what we currently do.

To date, the main findings of the Cochrane reviews have emphasised the limited number of RCT performed for many common dental interventions. The authors here highlight, however, that an absence of evidence should not to be interpreted as evidence for the lack of efficacy. In fact, all the research available demonstrates the advantages of PMC for restoring primary teeth. Furthermore, there is some evidence that suggest an enhanced success rate on teeth subjected to pulpotomy that are restored immediately with PMC. ${ }^{1}$

If all the textbooks, experts, academics and guidelines agree about the advantages of the use of PMC, why do primary care dental practitioners not use PMC routinely ${ }^{2}$ The authors suggest that this could be related to technical difficulties and funding issues. From an academic point of view, I think this is related to what we learn at Dental School. Indeed, as part of normal clinical training, Seddon report a mean of 8.1 restorations in glass ionomer or compomer and 1.9 in amalgam, compared with 0.2 using PMC, performed by undergraduates in UK for primary teeth. ${ }^{3}$ Reported use in clinical procedures by primary dental practitioners in the UK is $57 \%$ using glass ionomers, $35 \%$ amalgam and $8 \%$ PMCs. $^{2}$

The authors did not find any high-quality RCT upon which to base their analysis, so all the relevant information is contained in the abstract. Failure to use databases from Latin America and other regions may also be a potential source of bias. Although none of the reviews objectives were accomplished, this review can stimulate more and better research about the use of PMC in primary teeth. As Alderson and Roberts stated, "We should be willing to admit that we don't know so that the evidential base of health care can be improved for future generations." ${ }^{4}$ Even with improved evidence for the effectiveness of PMC we may still face a challenge in influencing the primary care practitioner.

\section{Sergio Uribe}

Department of Preventive and Paediatric Dentistry, Graduate School, Faculty of Dentistry, University of Valparaiso, Valparaiso, Chile

1. Farooq NS, Coll JA, Kuwabara A, Shelton P. Success rates of formocresol pulpotomy and indirect pulp therapy in the treatment of deep dentinal caries in primary teeth. Pediatr Dent 2000; 22:278-286.

2. Roshan D, Curzon ME, Fairpo CG. Changes in dentists' attitudes and practice in paediatric dentistry. Eur J Paediatr Dent 2003; 4:21-27.

3. Seddon RP. Undergraduate experience of clinical procedures in paediatric dentistry in a UK dental school during 1997-2001. Eur I Dent Educ 2004; 8:172-176.

4. Alderson P, Roberts I. Should journals publish systematic reviews that find no evidence to guide practice? Examples from injury research. Br Med J 2000; 32: 376-377.

Evidence-Based Dentistry (2007) 8, 10. doi:10.1038/sj.ebd.6400463x 